\title{
Development of an enzyme-based fiber optic biosensor for detection of haloalkanes
}

\begin{abstract}
Purpose - The main purpose of this study is to demonstrate the development of an enzymebased sensor for haloalkane detection. Haloalkane is a toxic compound that is found as genotoxic impurities in pharmaceutical products and contaminants in waste. The need to investigate the genotoxic level in pharmaceutical manufacturing is very crucial because of its toxicity effects on human health. The potential of mini protein as an alternative bioreceptor was explored with the aim to be more effective and stable under extreme conditions.

Design/methodology/approach - Mini proteins of haloalkane dehalogenase (HLD) were computationally designed and experimentally validated. Tapered multimode fiber (TMMF) was bio-functionalized with a bioreceptor either native (positive control) or mini protein. The absorbance-based sensor resulting from the binding interaction of mini protein with haloalkane was monitored through a wavelength range of 200-1,300 nm.

Findings - An increment of the UV absorption is observed at $325 \mathrm{~nm}$ when haloalkane interacted with the immobilized bioreceptors, native or mini protein. Both biosensors displayed a continuous response over the range of 5-250 $\mathrm{mM}$ haloalkane. They also had the capability to detect haloalkanes below $1 \mathrm{~min}$ and with an operational stability of up to seven days without significant loss of sensitivity.

Practical implications - The results indicate the potential viability of the enzyme HLD-based sensor to monitor the existence of haloalkane in both pharmaceutical and environmental products.

Originality/value - The paper describes an outcome of experimental work on TMMF-based biosensor coated with HLD for label-free haloalkane detection. Mini protein can be used as an effective bioreceptor with some structural modification to improve functionality and stability.
\end{abstract}

Keyword: Biosensor; Bioreceptor; Haloalkane dehalogenase; Mini protein 